\title{
ANTI-CRISIS POLICY OF THE LEADING CENTRAL BANKS AT THE PRESENT STAGE
}

\author{
MALKHAZ CHIKOBAVA \\ PhD in Economics, Associate Professor, \\ Ivane Javakhishvili Tbilisi State University, Georgia \\ malkhaz.chikobava@tsu.ge
}

Abstract. According to a review of "Central Banks: Monthly Balance Sheets", prepared by the consulting company Yardeni Research, Inc., the assets of the four leading central banks in the world - the US Federal Reserve (FED), the European Central Bank (ECB), the Bank of Japan and the People's Bank of China (PBOC), increased by 4.8 times over the specified period. Consequently, the liabilities of the big four central banks increased by 4.8 times. As it is known, the bulk of the Central Bank's liabilities is money issued by central banks (in the form of cash and deposits, on which commercial banks place their funds). Therefore, the money supply issued by four banks in a quarter of a century has also increased by 4.8 times.

The FED was especially distinguished by the fact that it began to sharply increase the issue of money in the midst of the financial crisis of 2008-2009. This was done under the flag of "quantitative easing" (QE). There were three "quantitative easing" programs, the last one ended in 2014. However, when the US declared a "pandemic" and began to impose quarantine restrictions, the Fed's printing press was again launched at full capacity. Now the Fed buys $\$ 120$ billion a month in the securities market $(\$$ 80 billion in US Treasury securities and another $\$ 40$ billion in mortgage securities). Accordingly, the dollar supply is increasing by the same amount every month.

The Bank of Japan is on the second place in terms of the growth rate of assets (and money supply). It began to build up assets and money supply earlier than other leading central banks, from the end of the 20th century to the beginning of the 21st century.

At the end of February 2021, the picture has changed radically. We see a rapid rise in the value of the indicator at the Bank of Japan, at the turn of 2018-2019. It crossed the $100 \%$ bar. Now its assets are equal to $127.4 \%$ of GDP. The indicators of the relative level of assets of the Fed and the ECB have grown very much. But the Central Bank of China has reduced the relative value of its assets by about a third. This is both the result of curbing the activity of the Chinese Central Bank and the fact that China has experienced high rates of GDP growth.

As for the other three leading central banks, their policy of increasing money supply will continue. The head of the US Federal Reserve, Jerome Powell, makes it clear that the US Central Bank, at least until the end of the year, will continue to buy securities in the same volumes (that is, $\$ 120$ billion a month).

ECB President Christine Lagarde said in March that the bank would accelerate asset purchases in the coming months under the Pandemic Emergency Purchase Program (PEPP). The volume of this program is 1.85 trillion EUR. In addition, the main ECB repurchase program continues to operate.

Other central banks that are not included in the survey by Yardeni Research, Inc. are also pursuing an asset buildup course. Among them are the Bank of England and the Swiss National Bank.

Leading central banks of the world, to justify the runaway money emission, refer to the "pandemic". The more money supply is created, the more likely it is that this currency will have a lower rate in relation to the competitor's currency. And the undervalued exchange rate of the national currency is a means of strengthening the international competitiveness of national business. However, such competition can lead to the collapse of the currencies of rival central banks.

KEYWORDS: NEGATIVE INTEREST RATE, PANDEMIC, VIRAL-ECONOMIC CRISIS, QUANTITATIVE EASING, CURRENCY WAR.

For citation: Chikobava, M., (2021). Anti-Crisis Policy of the Leading Central Banks at the Present Stage. Globalization and Business. 12, 143-151. (In Georgian). https://doi.org/10.35945/gb.2021.12.019 


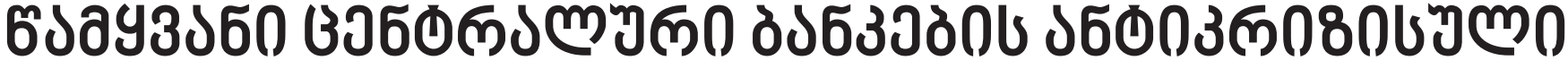

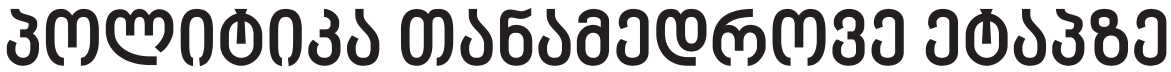

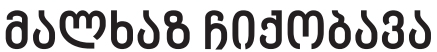

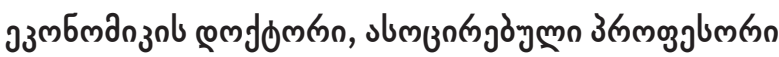

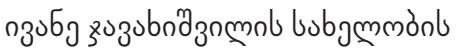

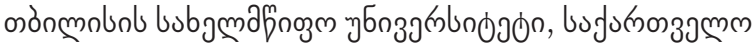

malkhaz.chikobava@tsu.ge

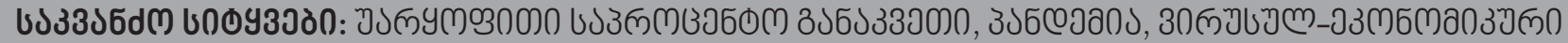

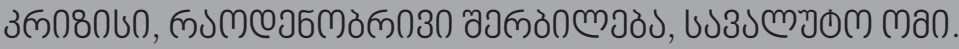

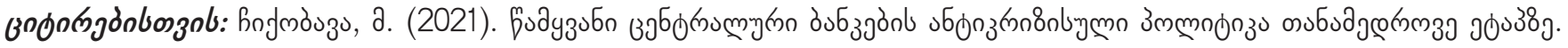

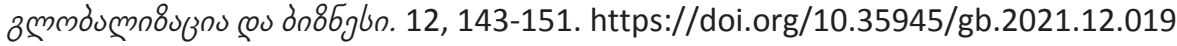

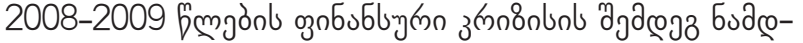

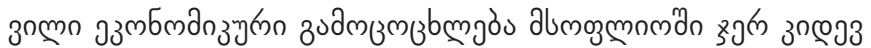

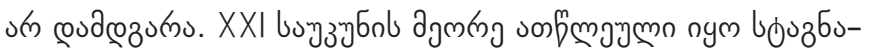

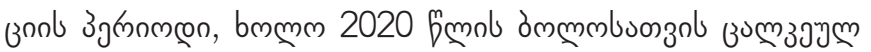
ปзэуб

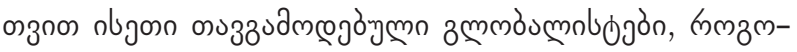

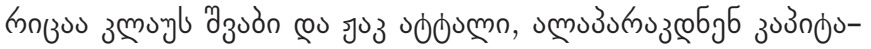

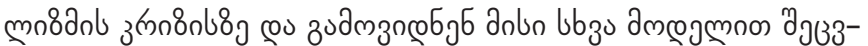
molu negnon (Katasonov, 2020).

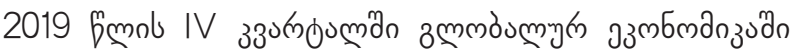

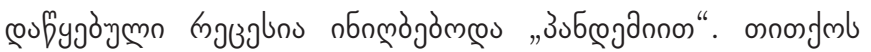

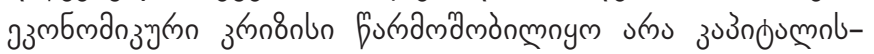

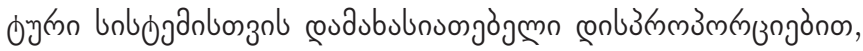

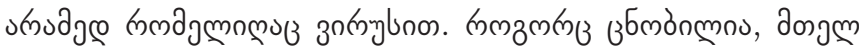

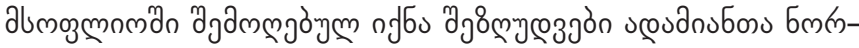

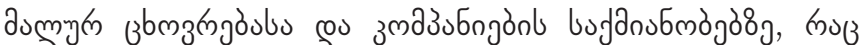

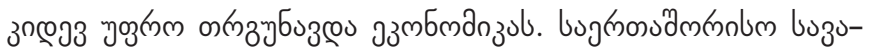

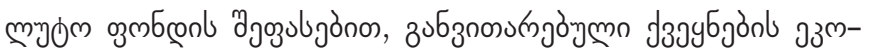

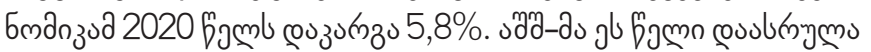

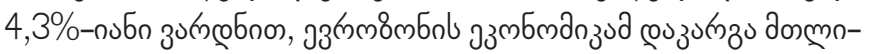
ง

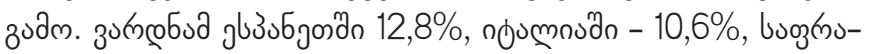

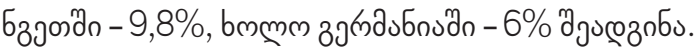

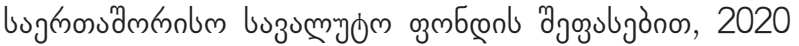

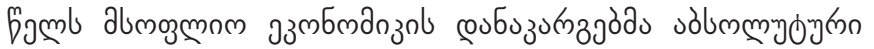

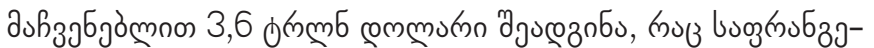

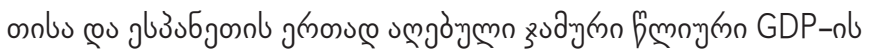

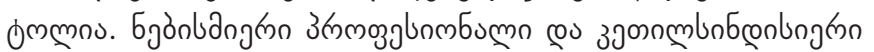

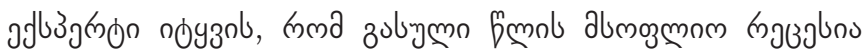

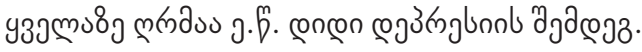

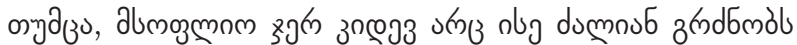

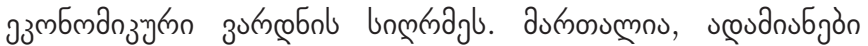

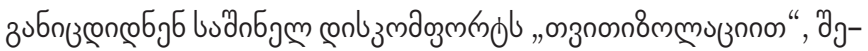

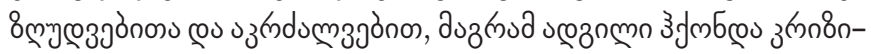

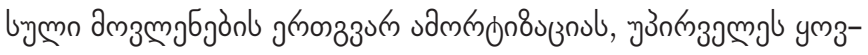

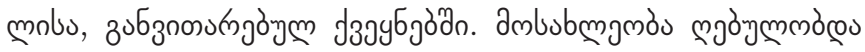

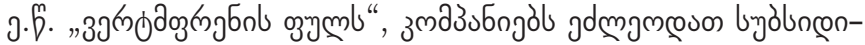

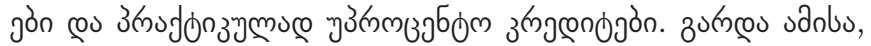

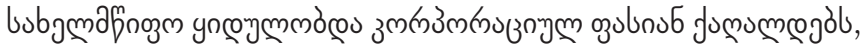

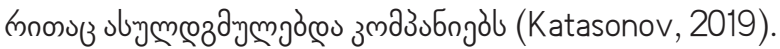

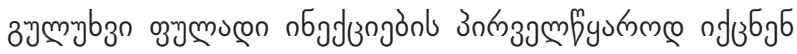

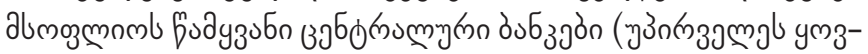
mobs, s.

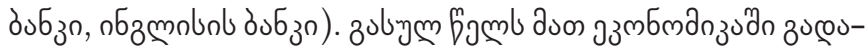

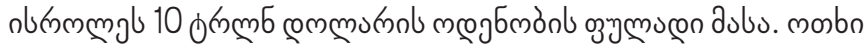

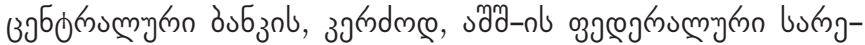

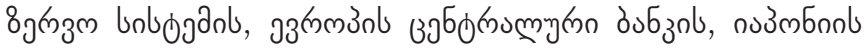

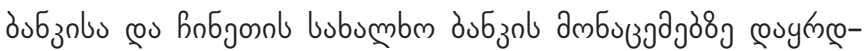

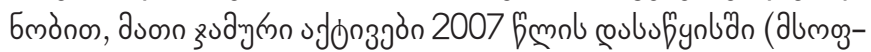

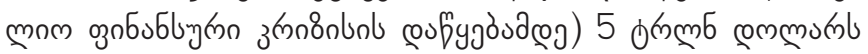

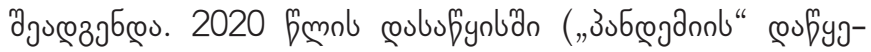

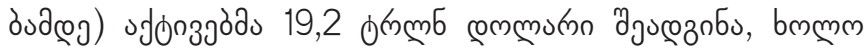

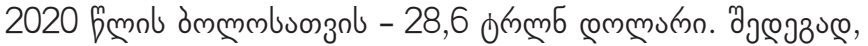

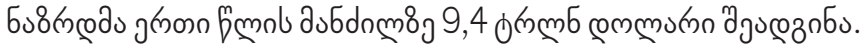

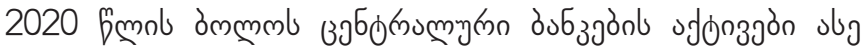

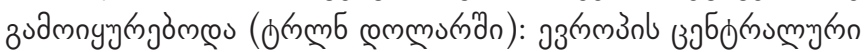
उuбz Uubumben du $\sigma_{3}$ - 5,9 (Bedianashvili, 2021).

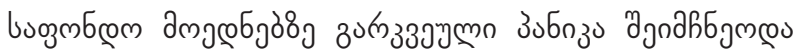
2020 bmol du 


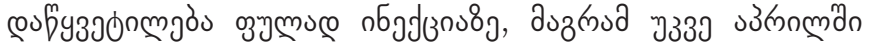

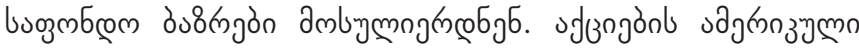

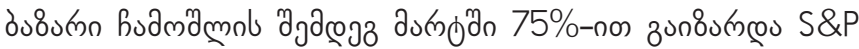
500 nбœ्व

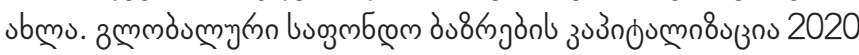

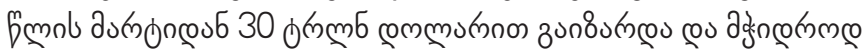

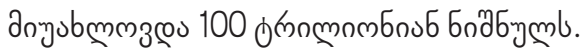

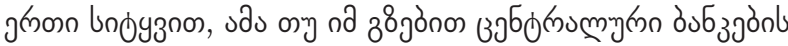

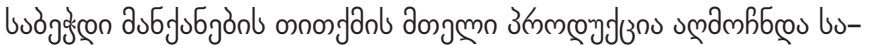

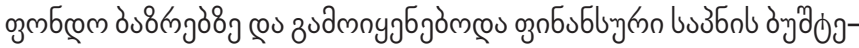

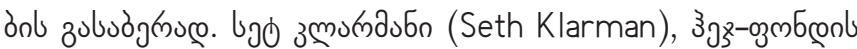

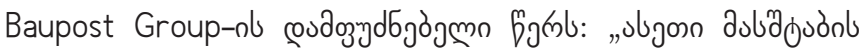

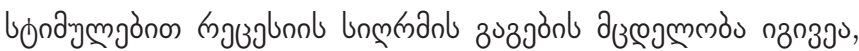

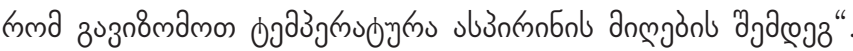

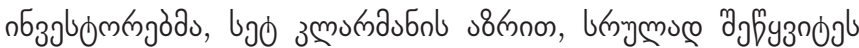

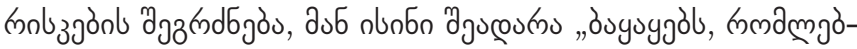

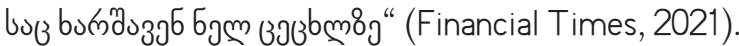

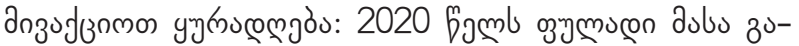

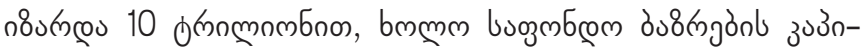

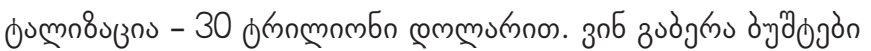

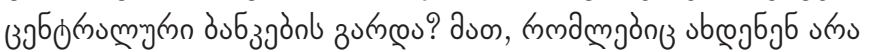

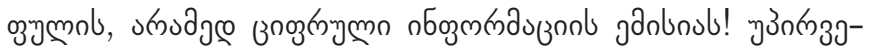

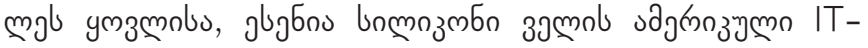

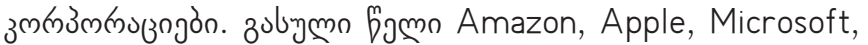

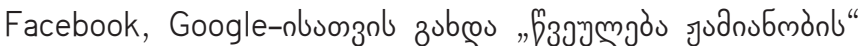

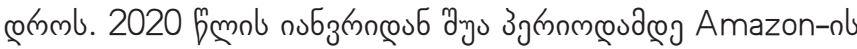

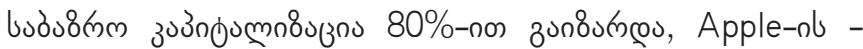
66\%-non, Microsoft-nl - 42\%-non, Facebook-nb - 40\%-nom,

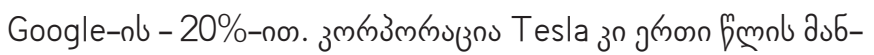

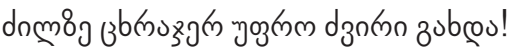

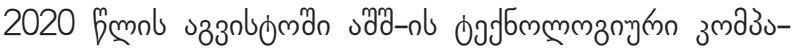

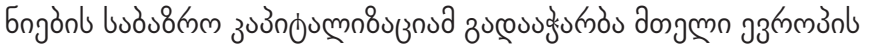

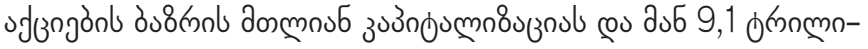

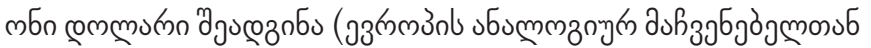

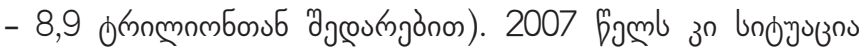

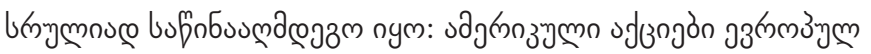

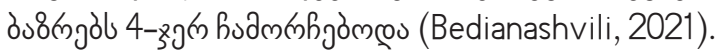

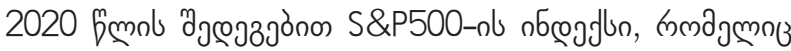

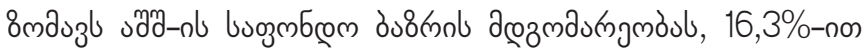

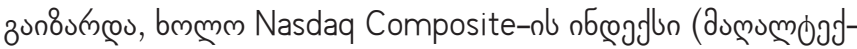

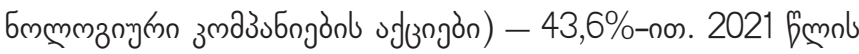

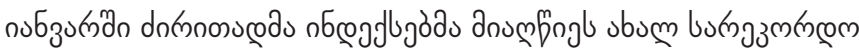

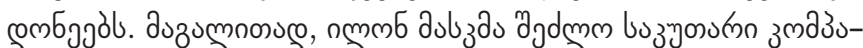

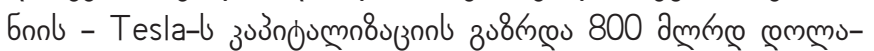

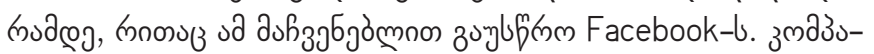

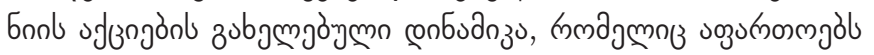

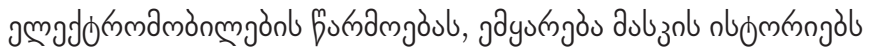
sbumn ззб

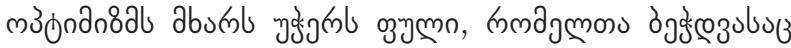

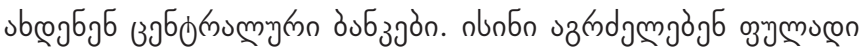

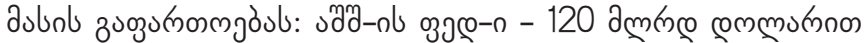

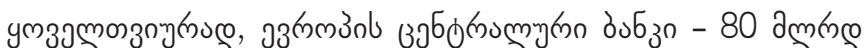
commumnon. Bank of America sogubjab, frma adju foayzub

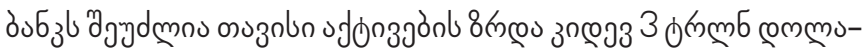

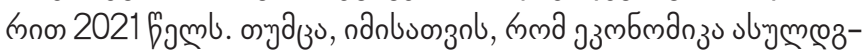

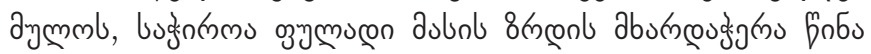

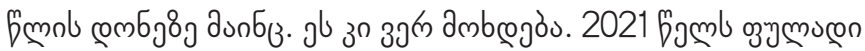

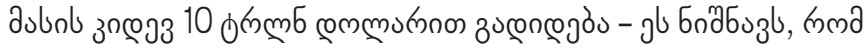

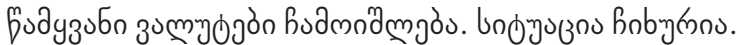

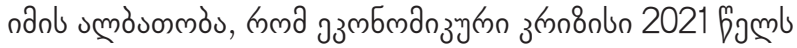

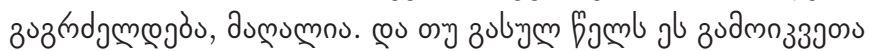

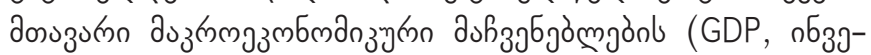

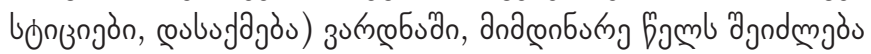

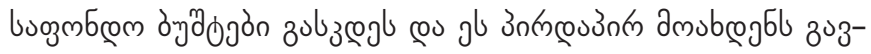

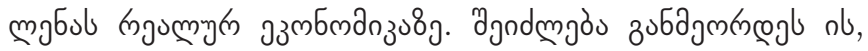

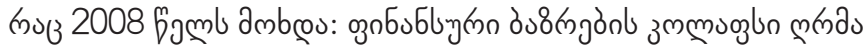

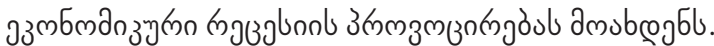

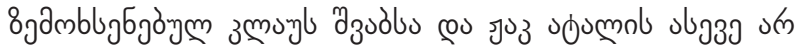
uzgnum, mma z rno

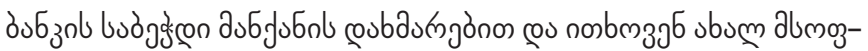

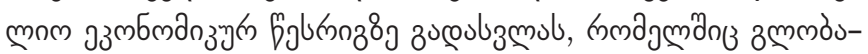
mynn jmodnl azuz

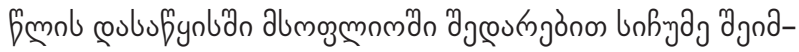

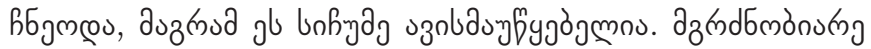

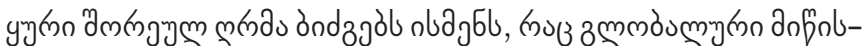

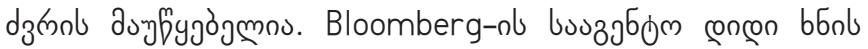

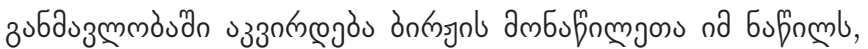

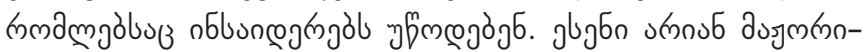

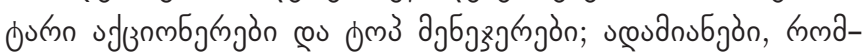

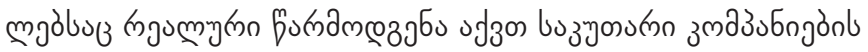

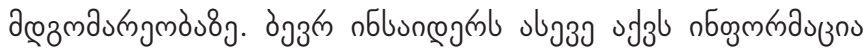

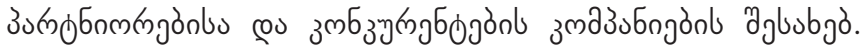

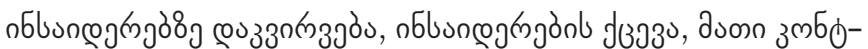

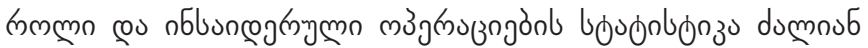

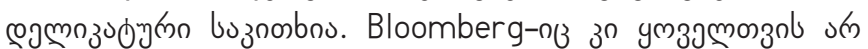

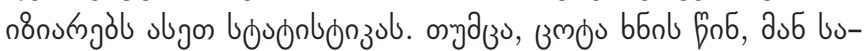

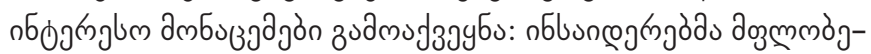

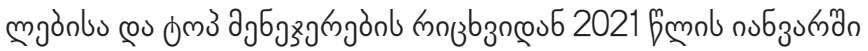

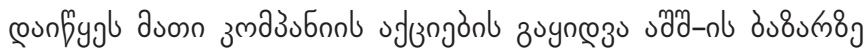

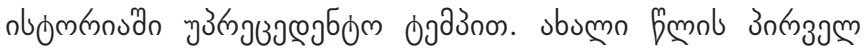

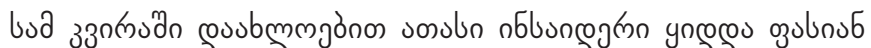

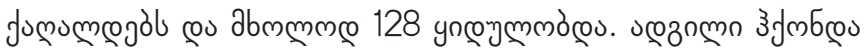

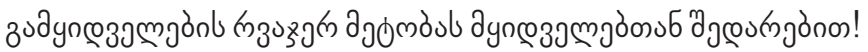

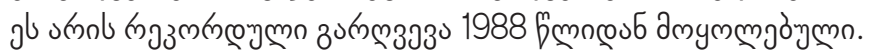

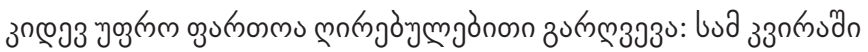

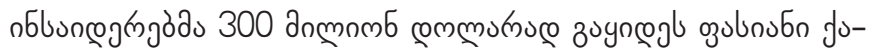

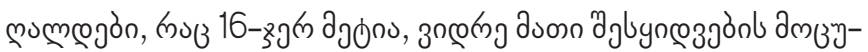
mmòv (Durden, 2021).

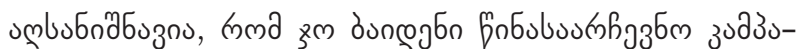

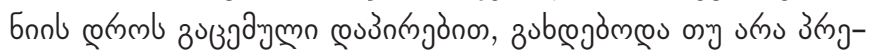




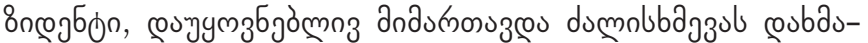

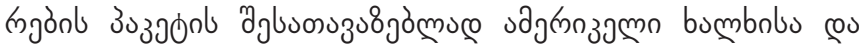

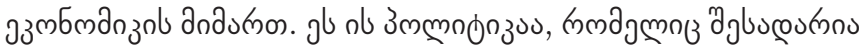

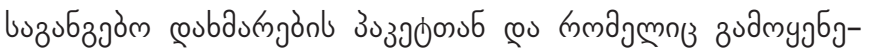

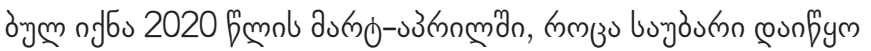

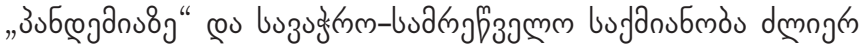

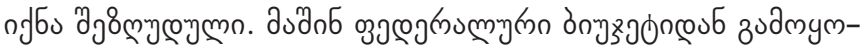

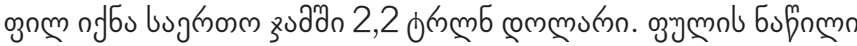

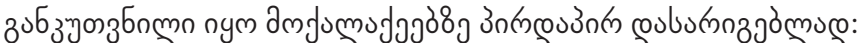

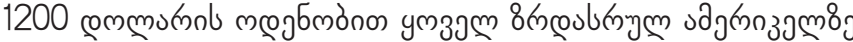

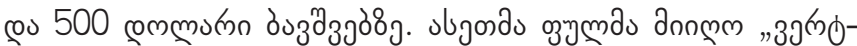

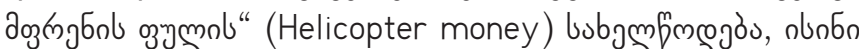

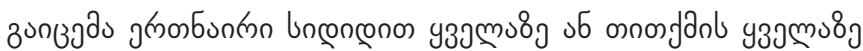

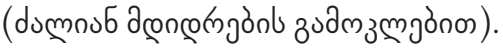

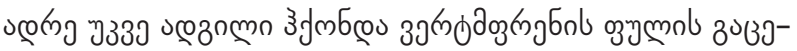

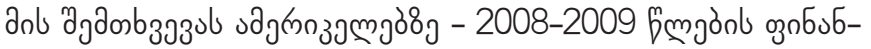

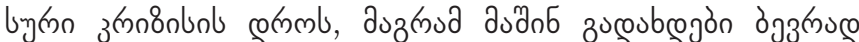

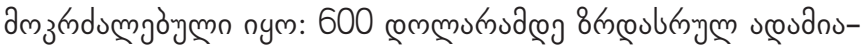

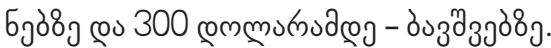

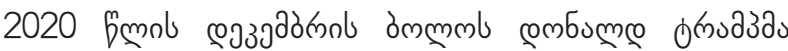

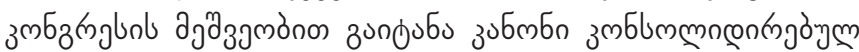
unzagjojobog (Consolidated Appropriations Act - CAA),

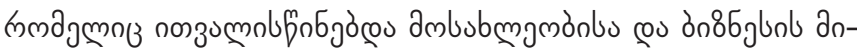

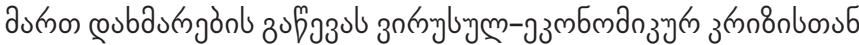

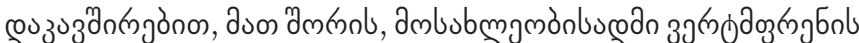

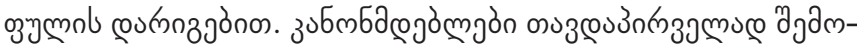

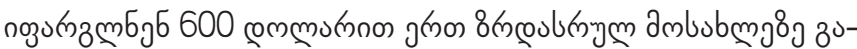

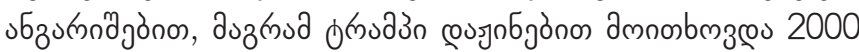

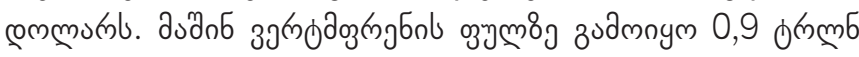
commumo (Papava \& Charaia, 2021).

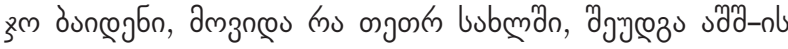
змб

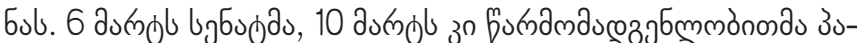

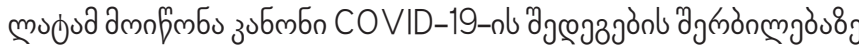
(COVID (Covid Relief Act, CRA), amubonobs ho dob ringumn-

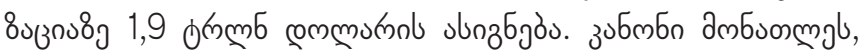

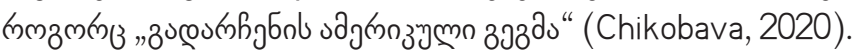

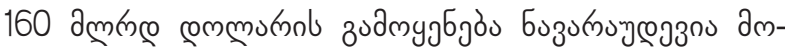

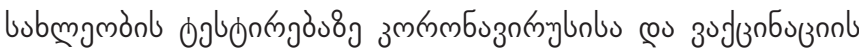

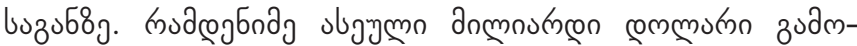

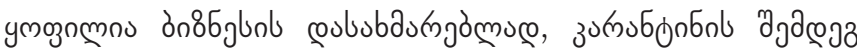

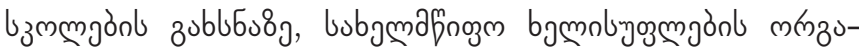

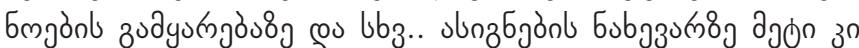

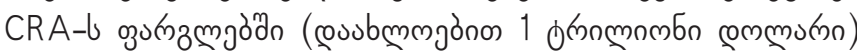
- zuбzचnos

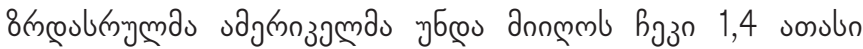

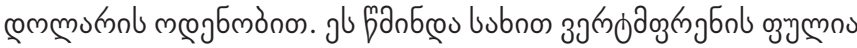

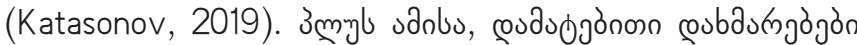

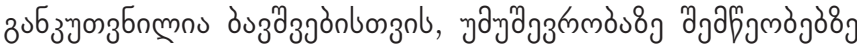

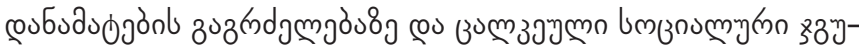

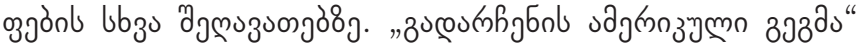

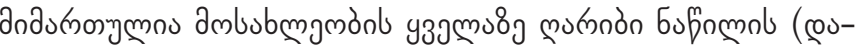

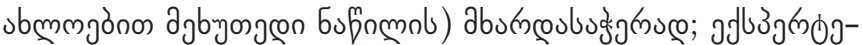

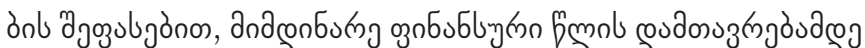

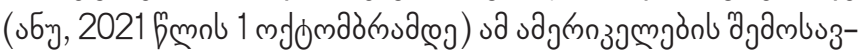

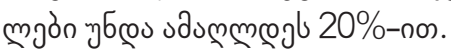

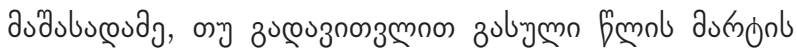

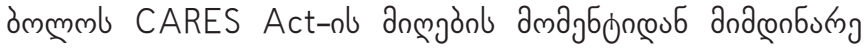

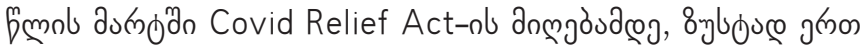

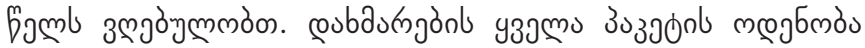

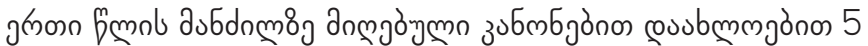

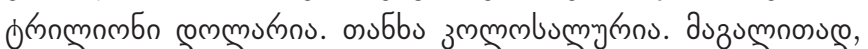

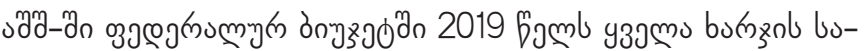

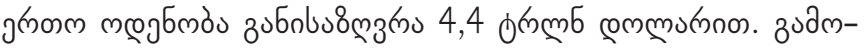

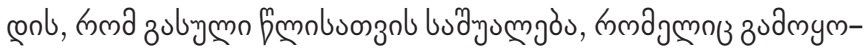

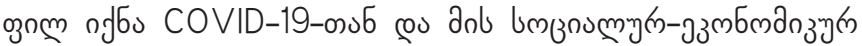

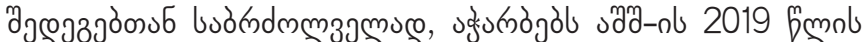

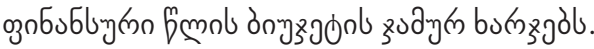

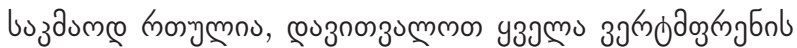

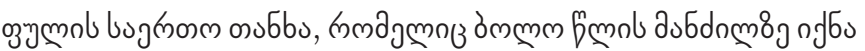

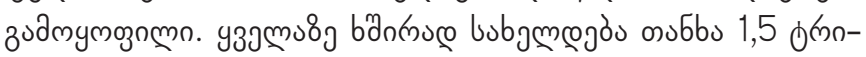

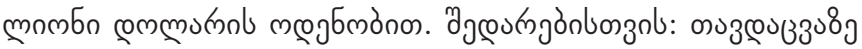

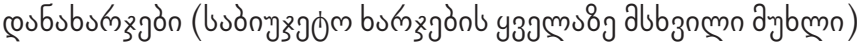

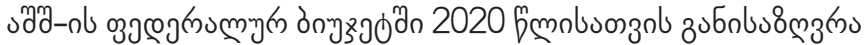
738 amme communnol megбmònon.

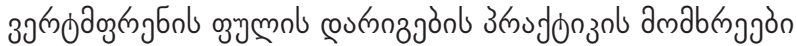

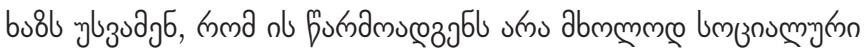

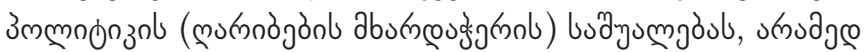

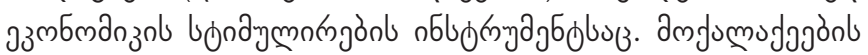

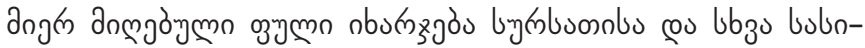

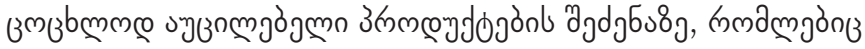

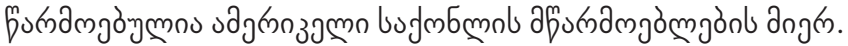

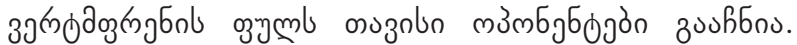

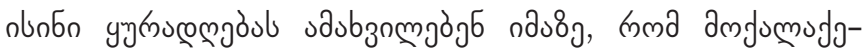

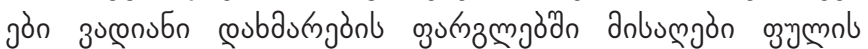

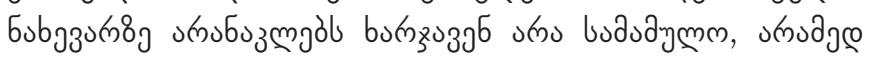

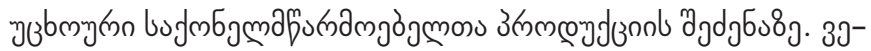

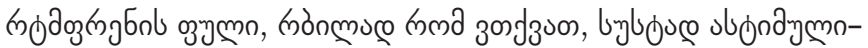

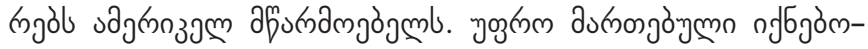

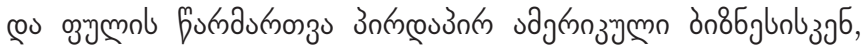

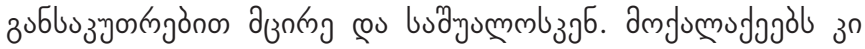

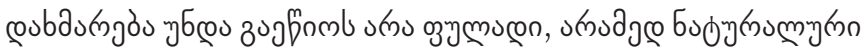
bubnon.

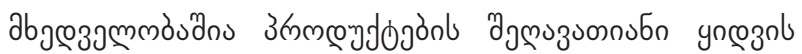
उஙnmzrnodo (Supplemental Nutrition Assistance Program),

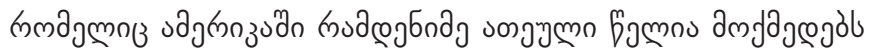

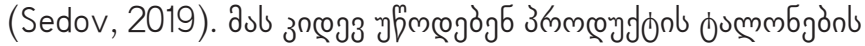

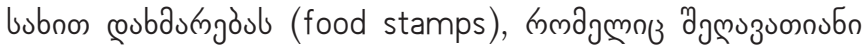

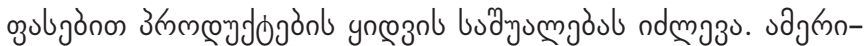

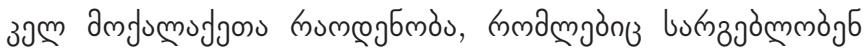




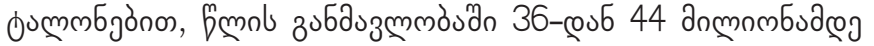

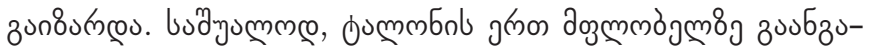

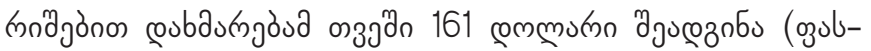

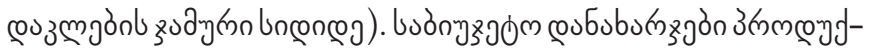

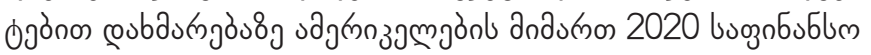

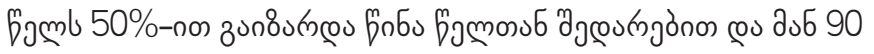

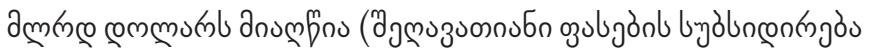

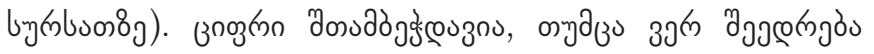

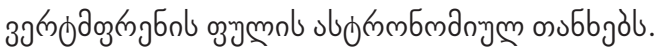

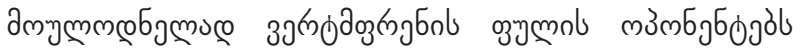

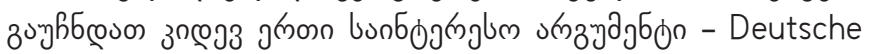

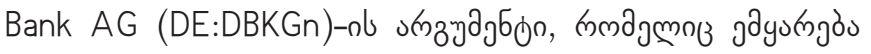

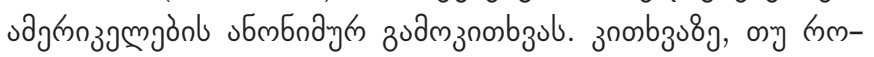

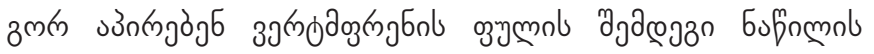

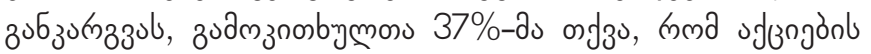

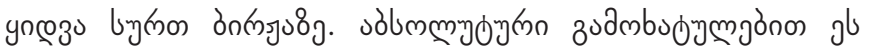

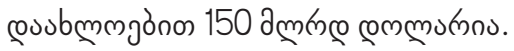

$30 \sigma_{3}$ JP Morgan Chase- -

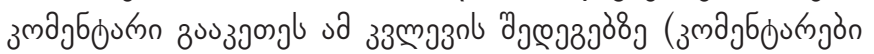

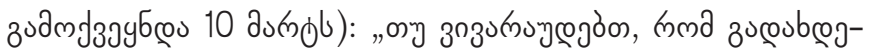

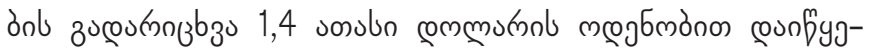

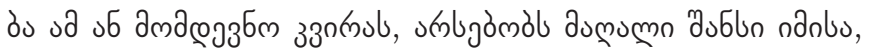

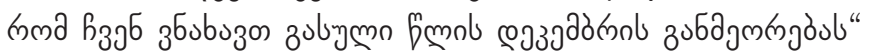

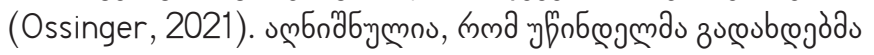

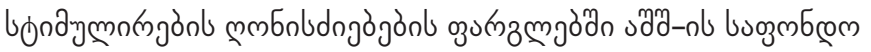

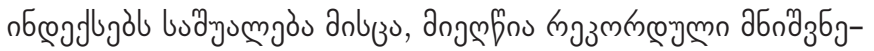

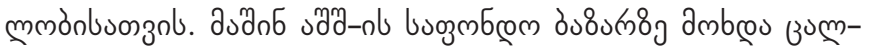

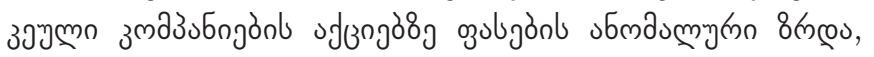

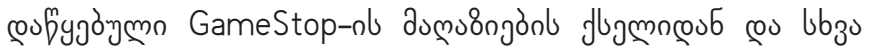

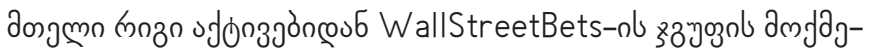

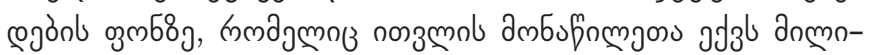

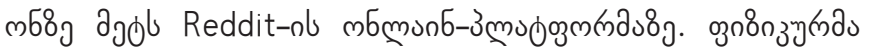

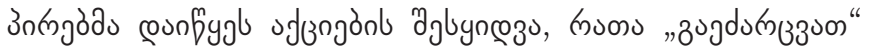

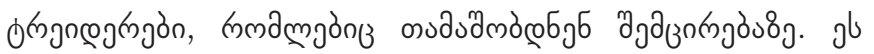

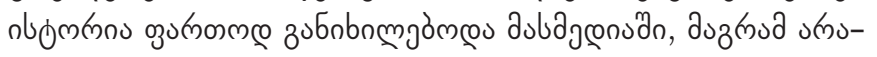

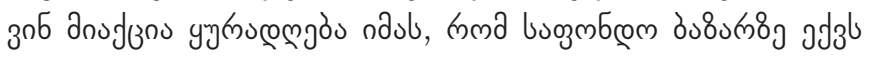

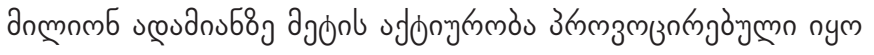

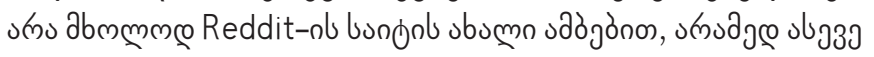

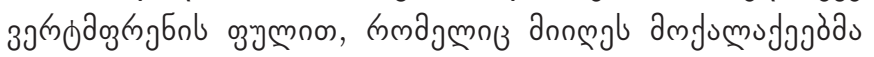

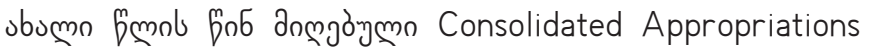
Act-nl anbgeznon.

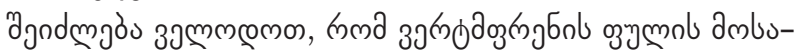

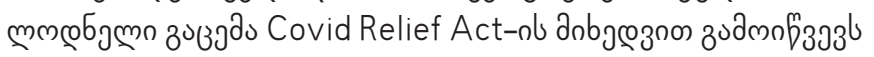

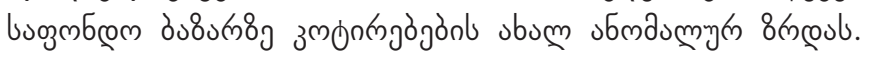

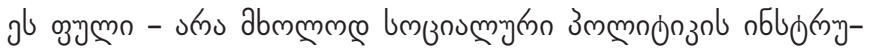

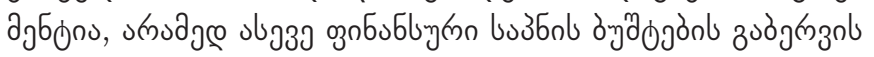

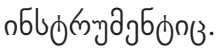

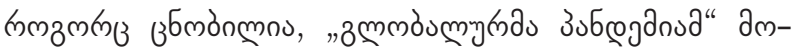

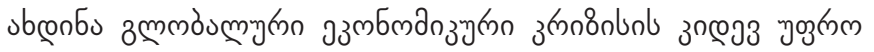
зง

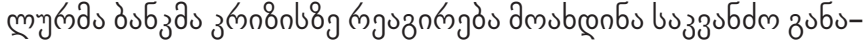

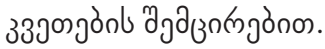

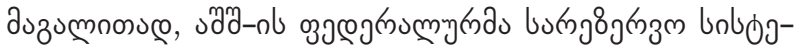

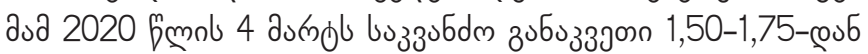

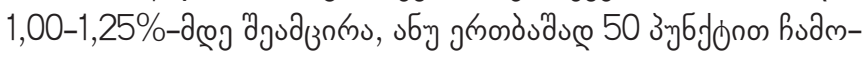

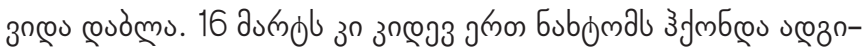

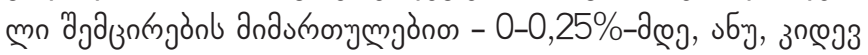

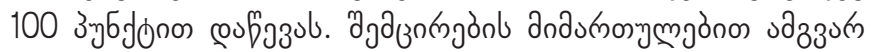

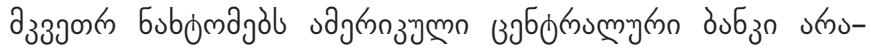

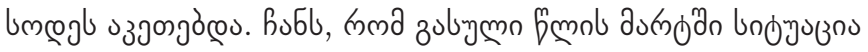

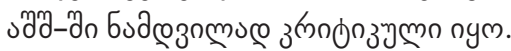

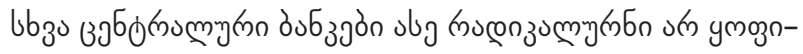

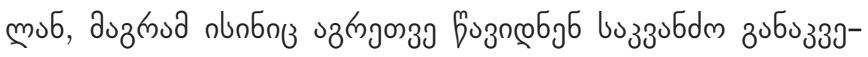

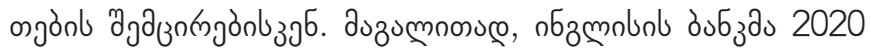

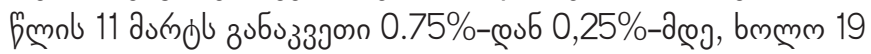

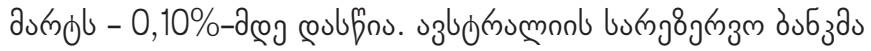

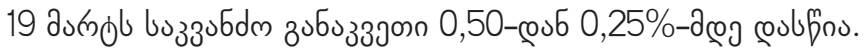

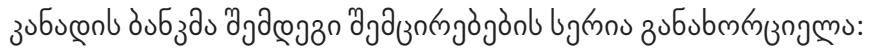

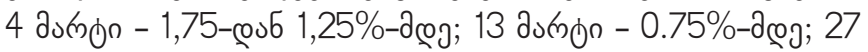
aง

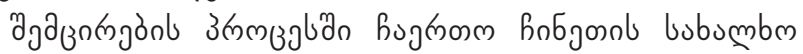

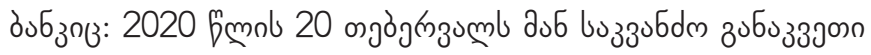

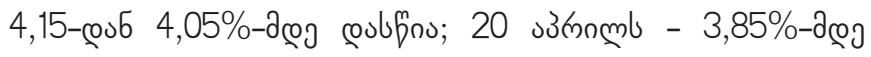
(Chikobava, 2020).

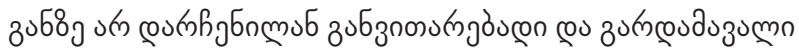

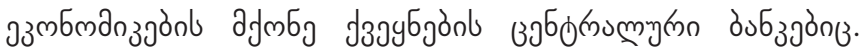
उ nus

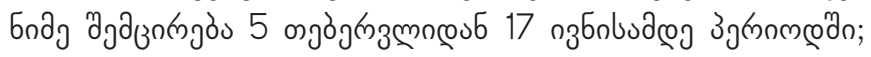

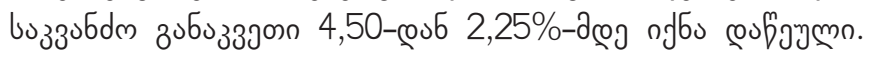

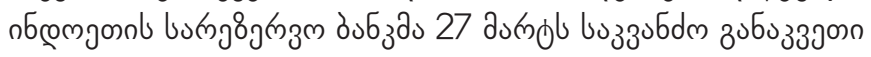

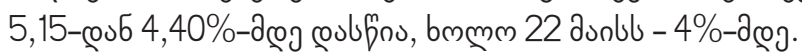

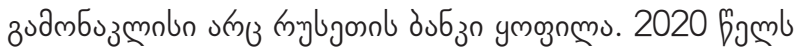

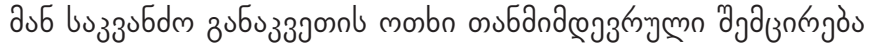

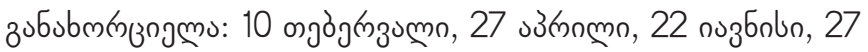

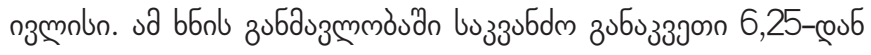
4,25\%-деg солgajo.

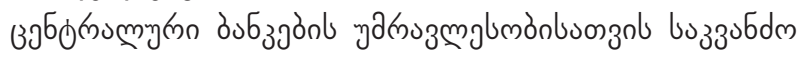

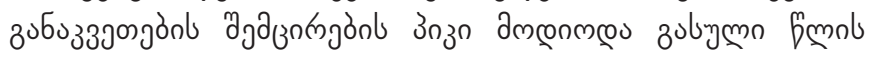

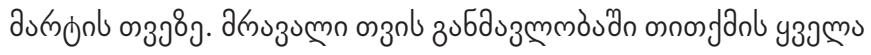

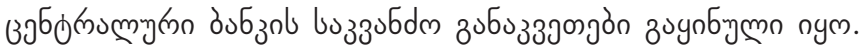

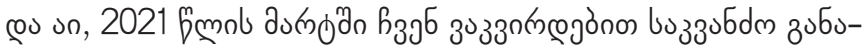

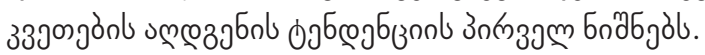

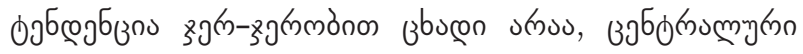

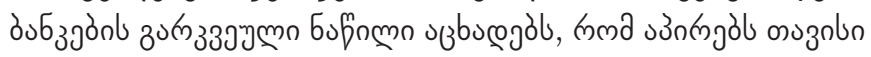

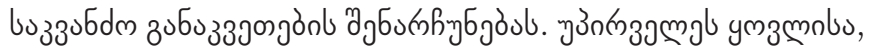

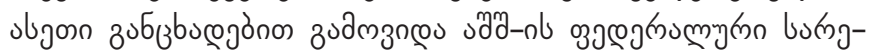

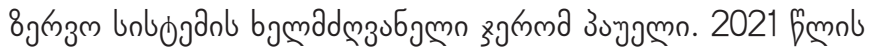

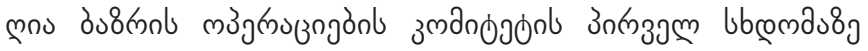

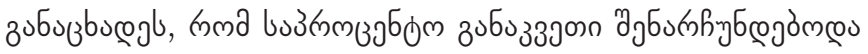

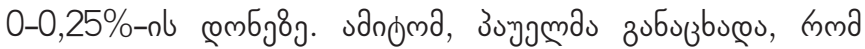




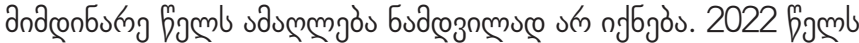

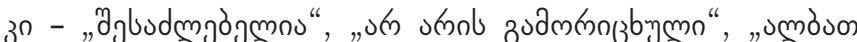

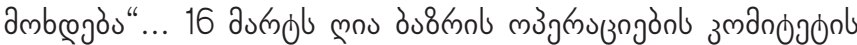

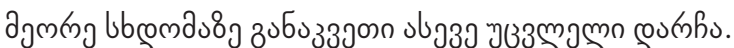

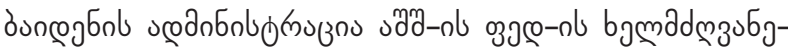

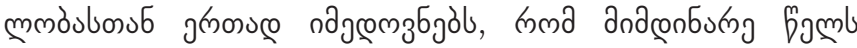

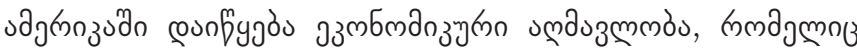

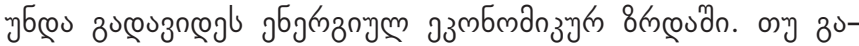

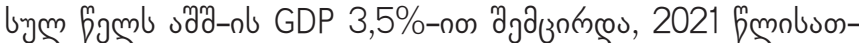

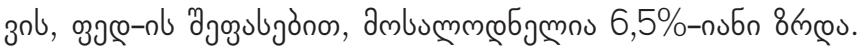
амmмmलnб

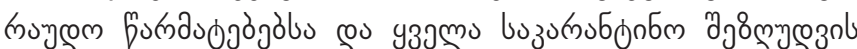

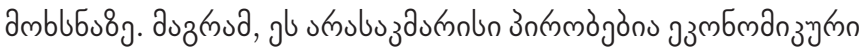
zodmzimizmmànbsonzol.

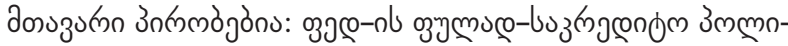

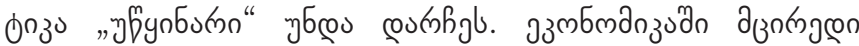

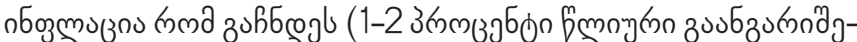

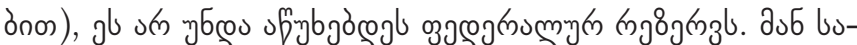

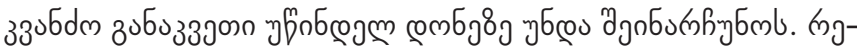

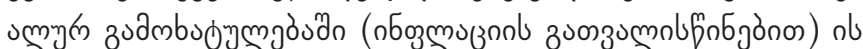

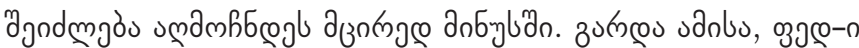

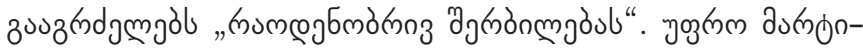

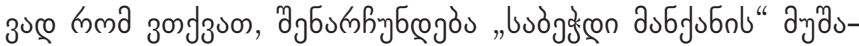

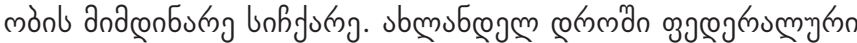

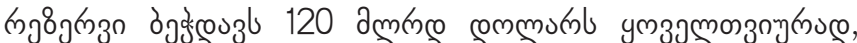

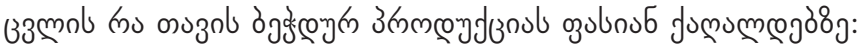

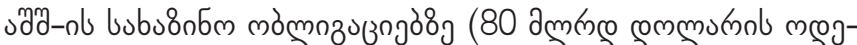

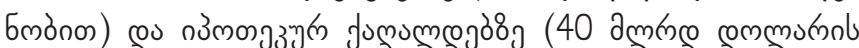

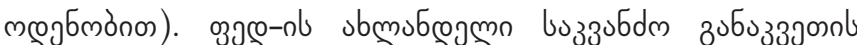

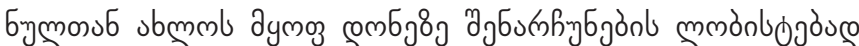

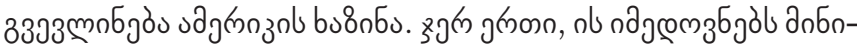

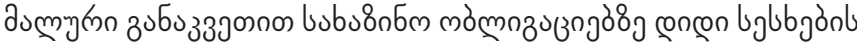

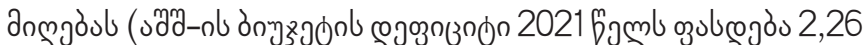

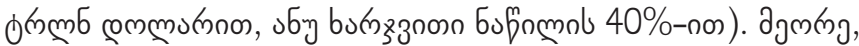

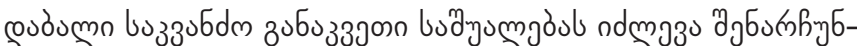

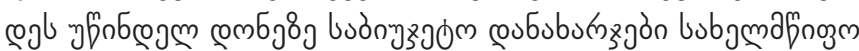
zumnl amalusbyngasuog.

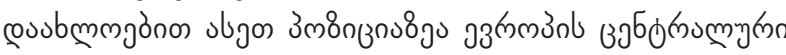

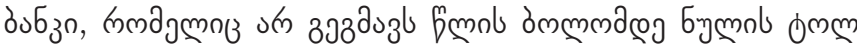

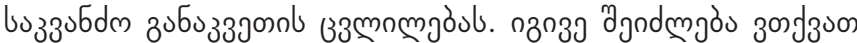

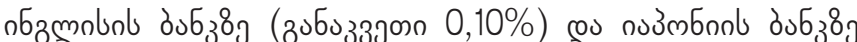

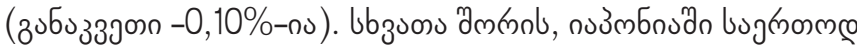

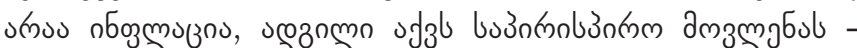
egogmoziol.

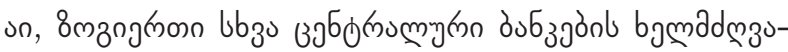

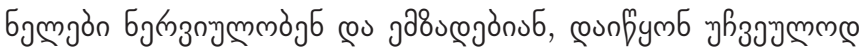

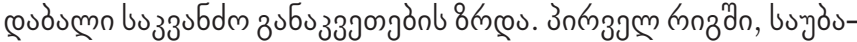

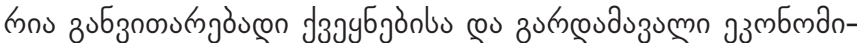

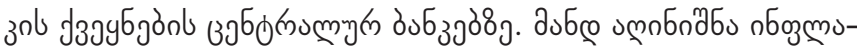

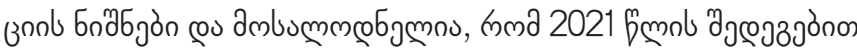

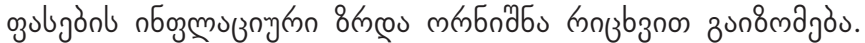

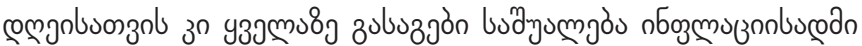

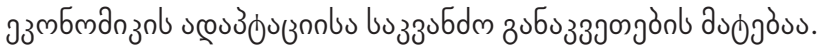

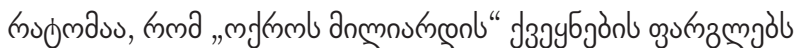

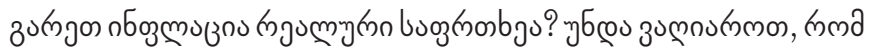

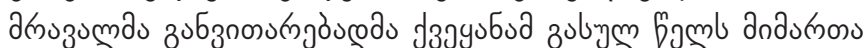

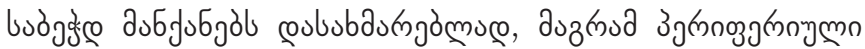

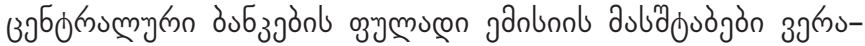

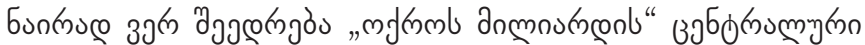

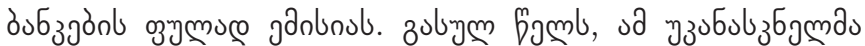

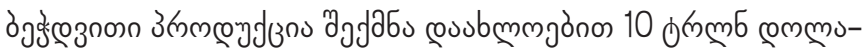

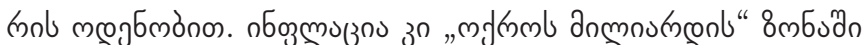
anбnaummmno. muano budag?

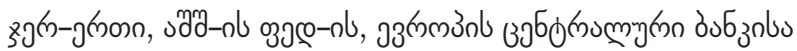

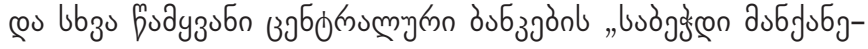

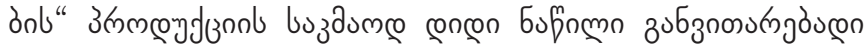

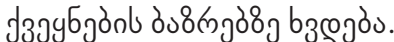

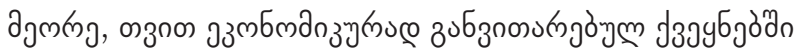

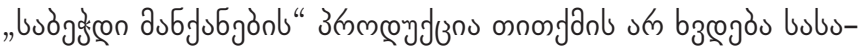

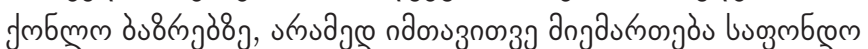

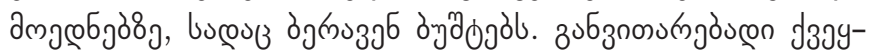

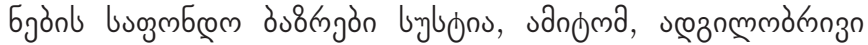

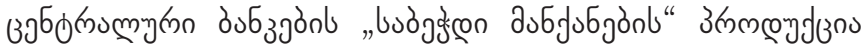

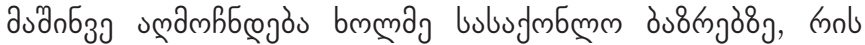

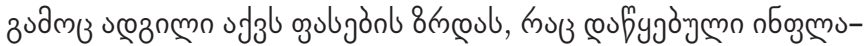

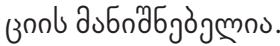

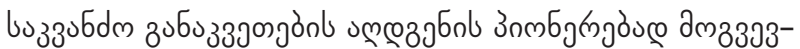

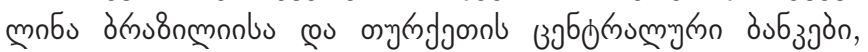

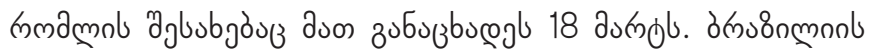

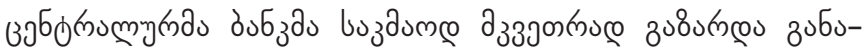

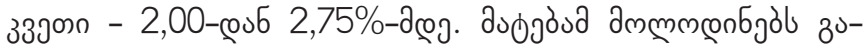

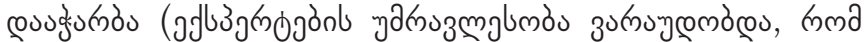

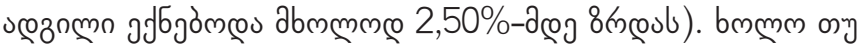

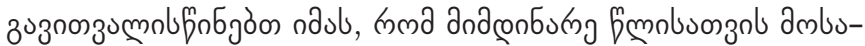

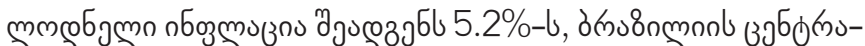

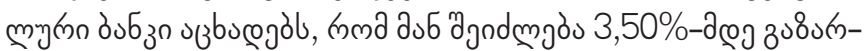
comb zo6s 33900 .

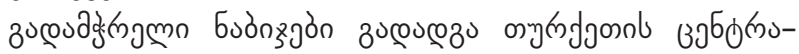

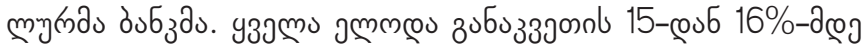

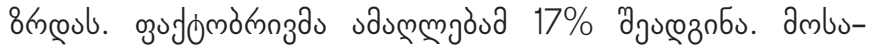

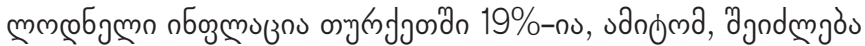

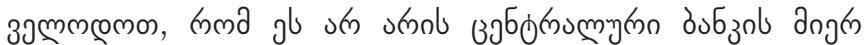

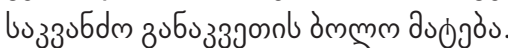

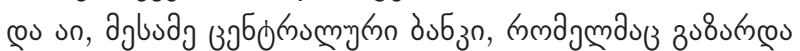

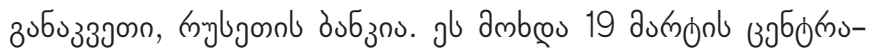

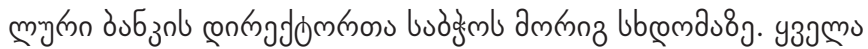

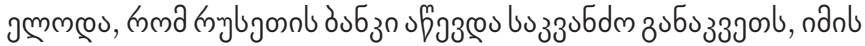

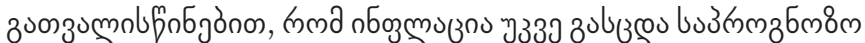

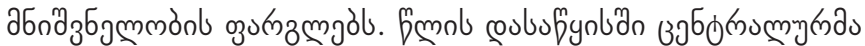

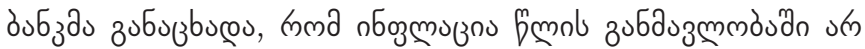




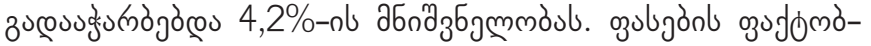

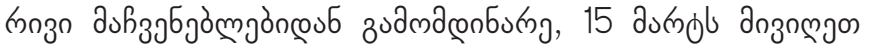

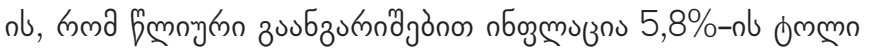

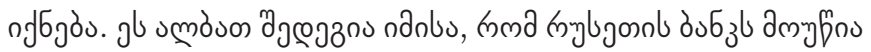

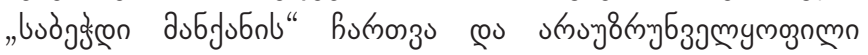

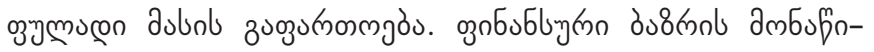

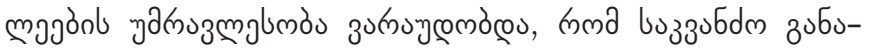

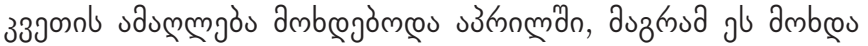

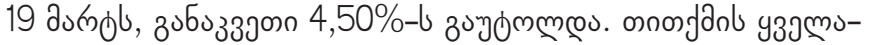

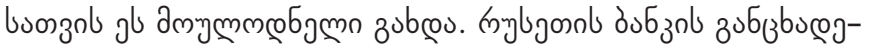

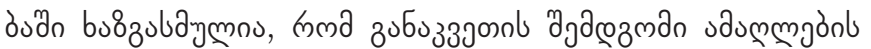

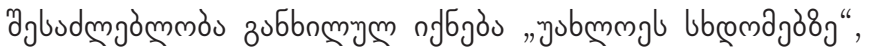

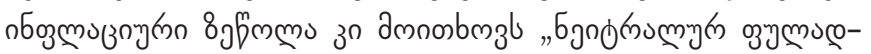

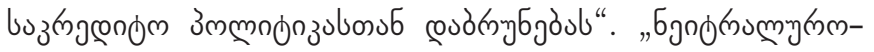

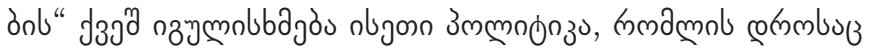

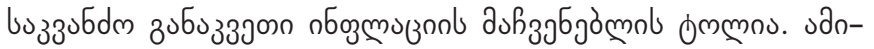

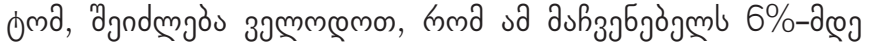

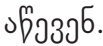

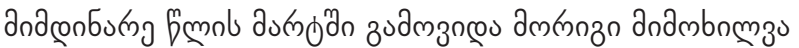

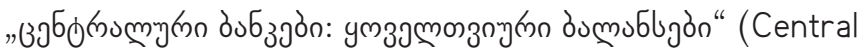

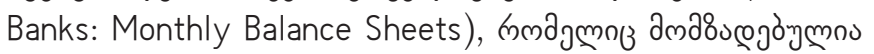

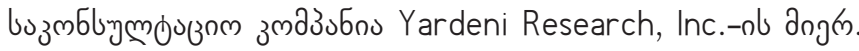

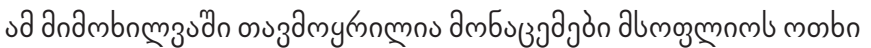

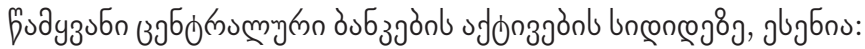

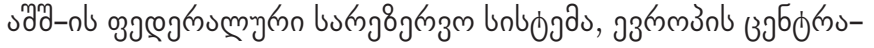

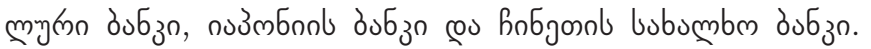

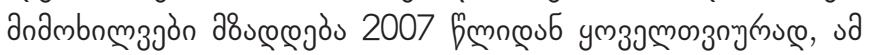

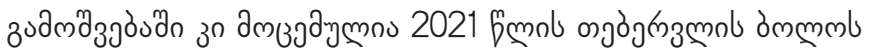
ambusjajgòn (Yardeni and Quintana, 2021).

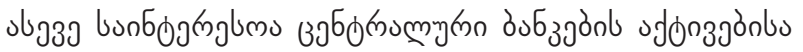

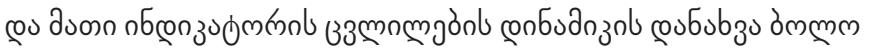

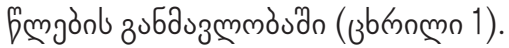

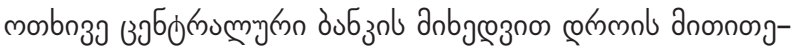

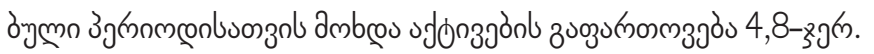

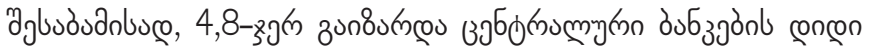

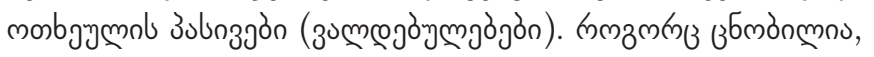

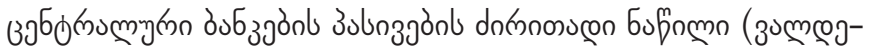

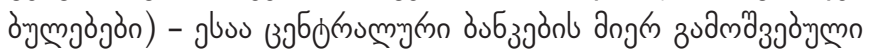

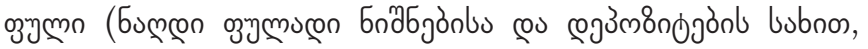

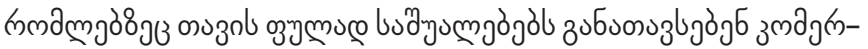

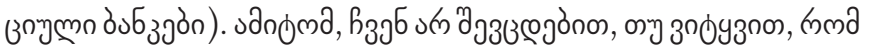

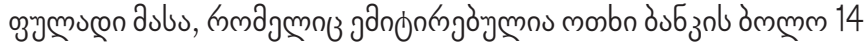

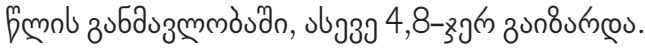

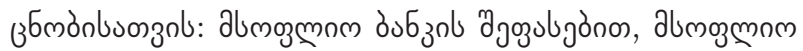

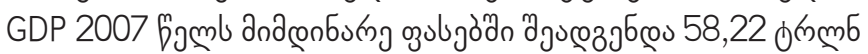

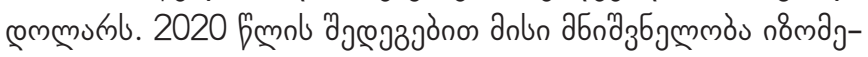

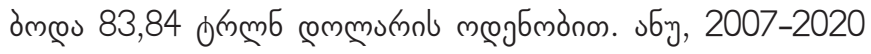

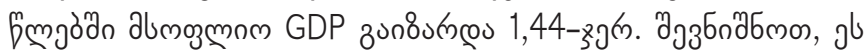

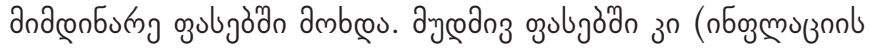

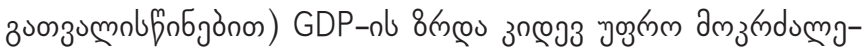

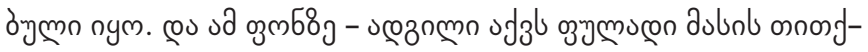

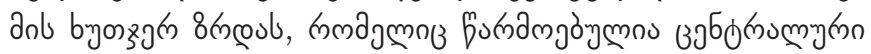

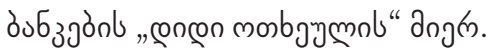

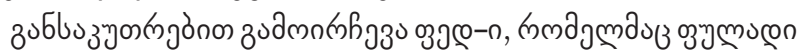

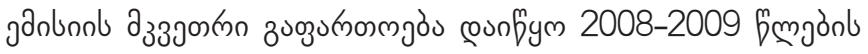

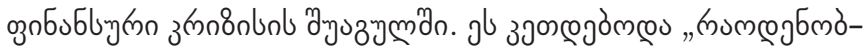

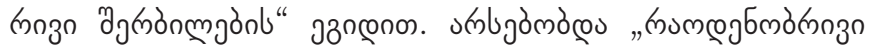

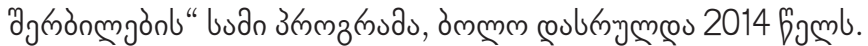

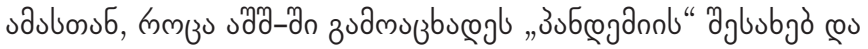

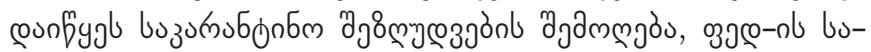

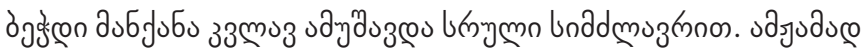

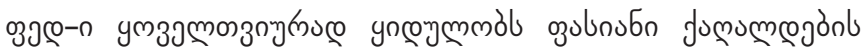
3.80

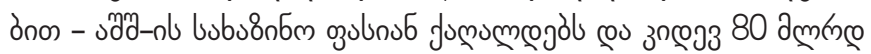

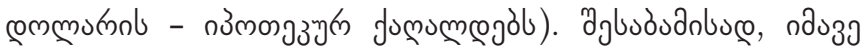

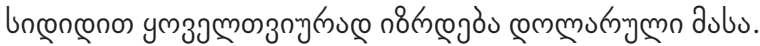

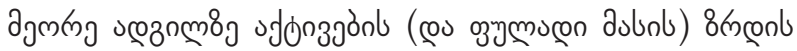

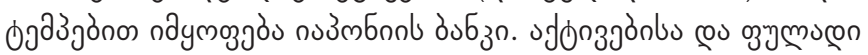

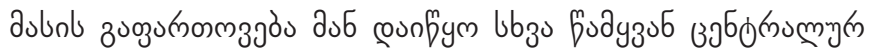
उง coubufynoton.

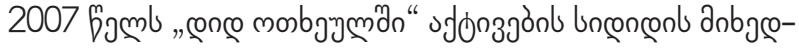

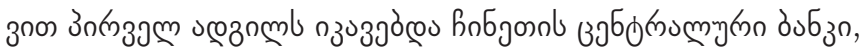

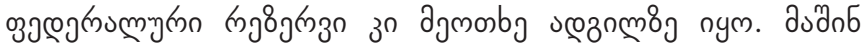

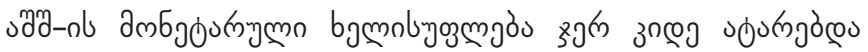

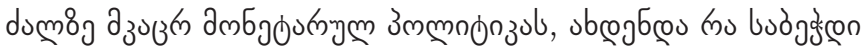

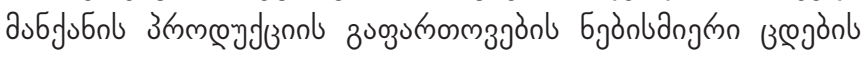

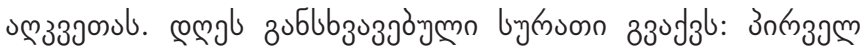
ง

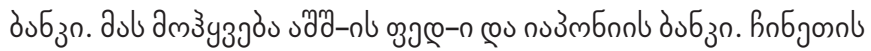

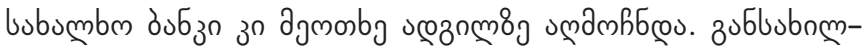

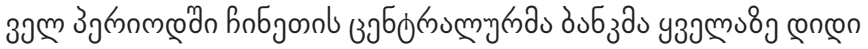

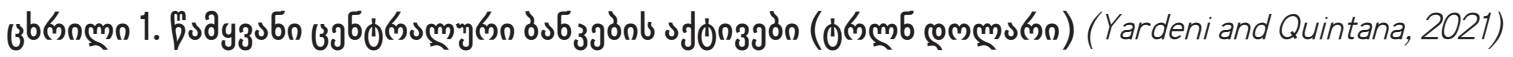

\begin{tabular}{|c|c|c|c|}
\hline 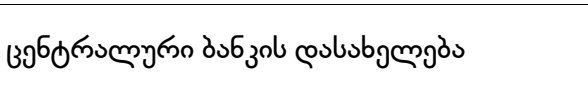 & 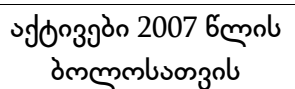 & 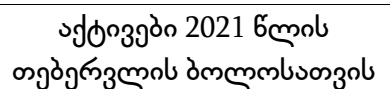 & 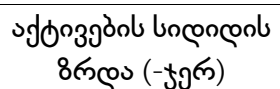 \\
\hline 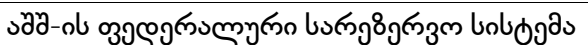 & 0,8 & 7,6 & 9,50 \\
\hline 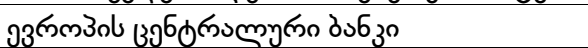 & 1,9 & 8,6 & 4,53 \\
\hline nu3mbnol du6 30 & 1,0 & 6,8 & 6,80 \\
\hline Robgosol lubumbm dub zo $^{\circ}$ & 2,3 & 5,9 & 2,57 \\
\hline jogn & 6.0 & 28,8 & 4,80 \\
\hline
\end{tabular}




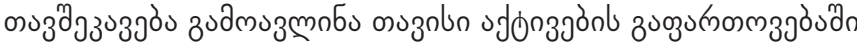

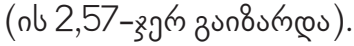

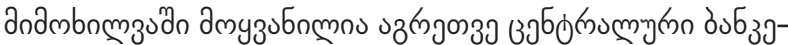

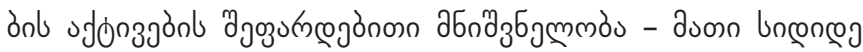

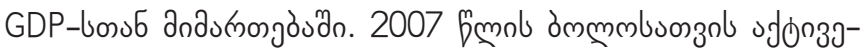

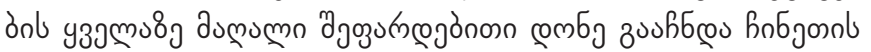

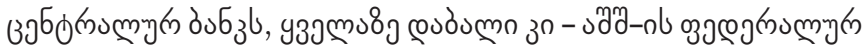
bumgigngुm lnbogaul.

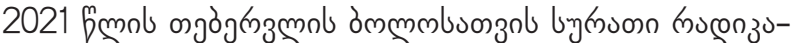

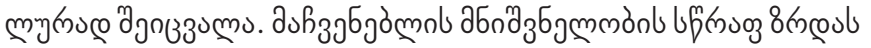

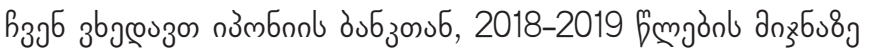

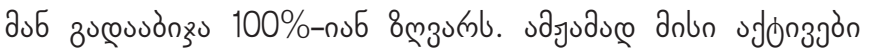

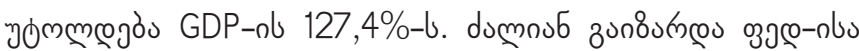

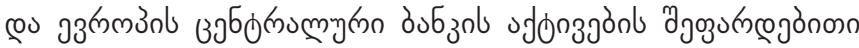

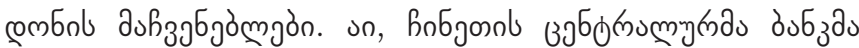

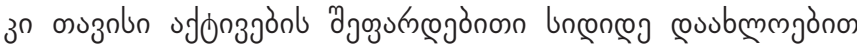
agbuagenon aguazotru.

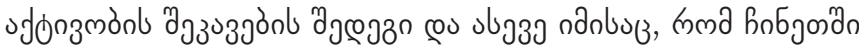

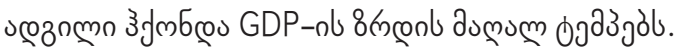

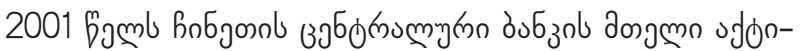

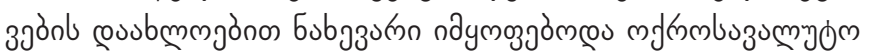

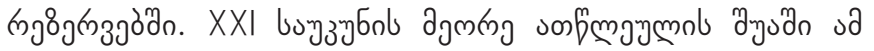

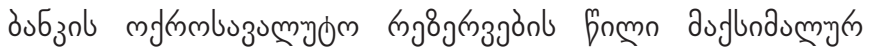

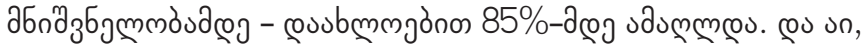
2021 pmol o क

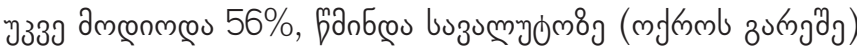

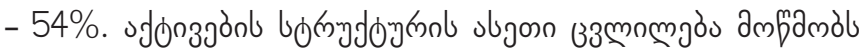

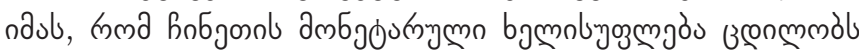

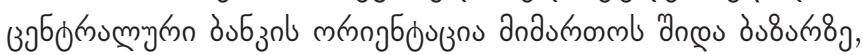

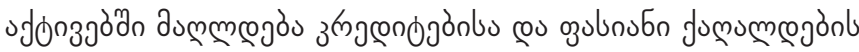

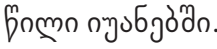

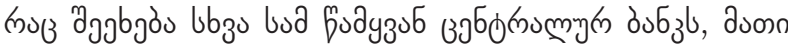
зु

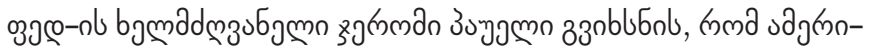

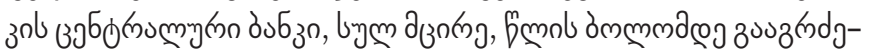

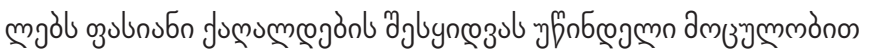
( ১б

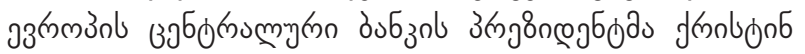

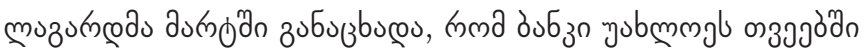

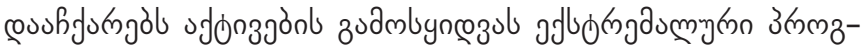
nodob - PEPP (Pandemic Emergency Purchase Programme)

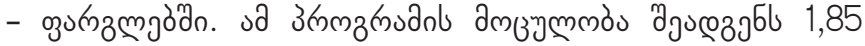

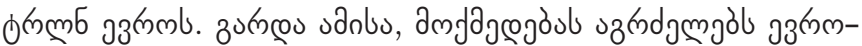

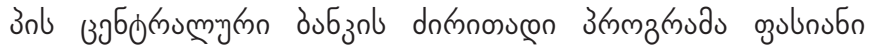

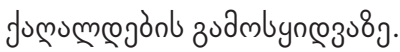

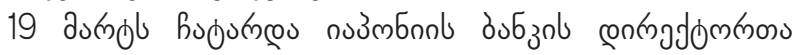

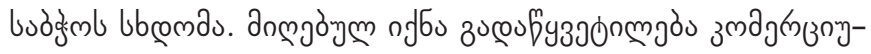

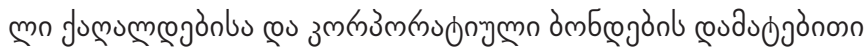

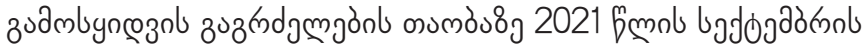

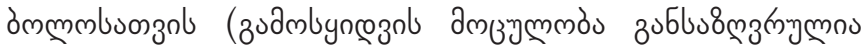

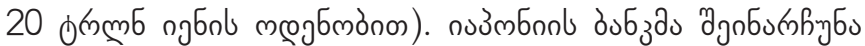

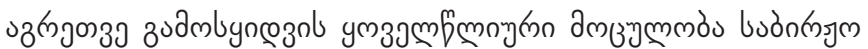

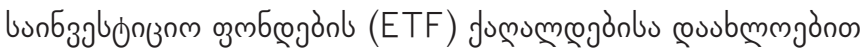
12 j б

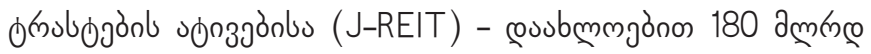

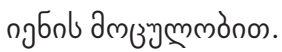

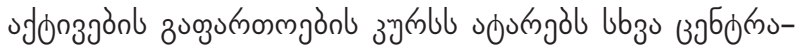

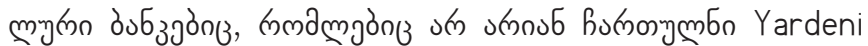

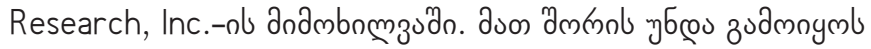
nб

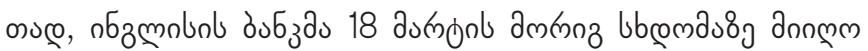

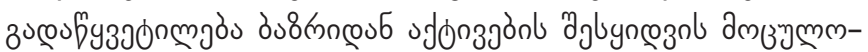

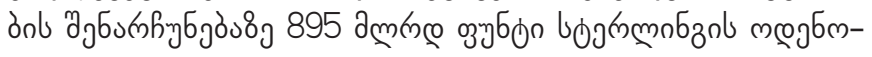

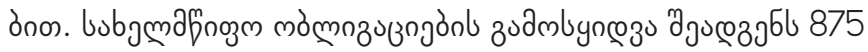

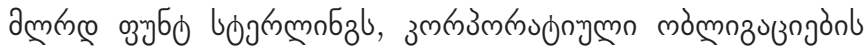

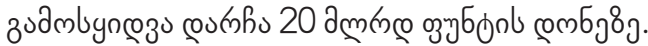

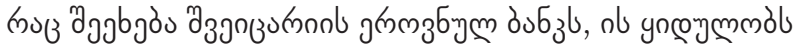

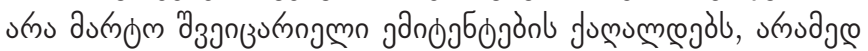

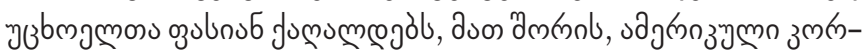
उmmozojónbul.

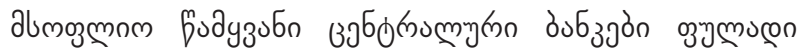

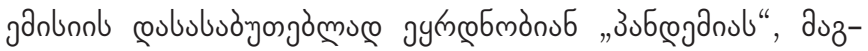

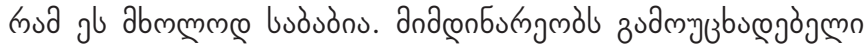

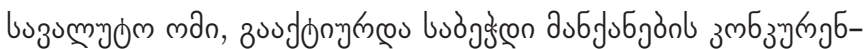

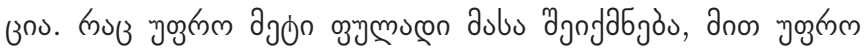

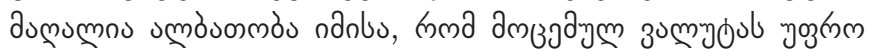
œ

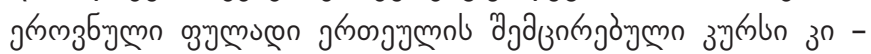

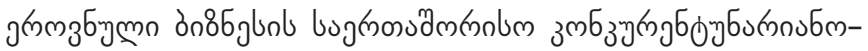

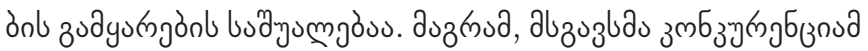

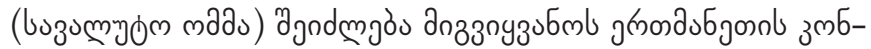

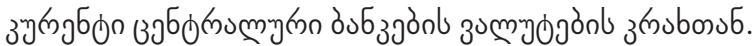

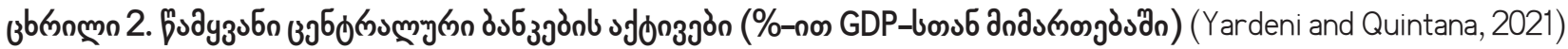

\begin{tabular}{|c|c|c|}
\hline 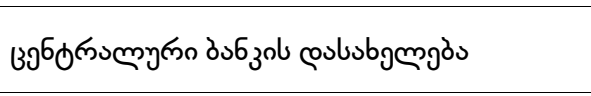 & 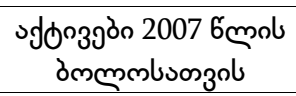 & 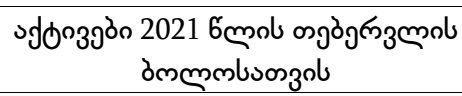 \\
\hline 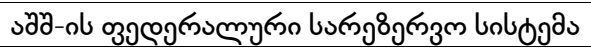 & 6 & 33,4 \\
\hline 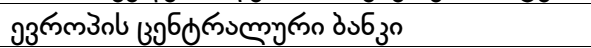 & 12 & 59,5 \\
\hline nu3m6nol $3063^{\circ}$ & 21 & 127,4 \\
\hline ho6josol bubu mbe dub & 53 & 35,1 \\
\hline
\end{tabular}




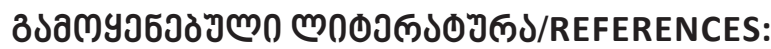

Bedianashvili, G. (2021). Macroeconomic and Cultural Determinants of the COVID-19 Pandemic Crisis. Bulletin of the Georgian National Academy of Sciences. 15(2), 191-197.

Bedianashvili, G. (2021).The Socio-Economic Effects of Covid-19 and the Challenges of Economic Uncertainty. Materials of VI International Scientific Conference: Challenges of Globalization in Economics and Business. Ivane Javakhishvili Tbilisi State University, Economics and Business Faculty, 34-38 (In Georgian).

Chikobava, M. (2020). Covid - 19 as a Form of Hybrid War. Globalization and Business, 10. 79-85. (In Georgian). https://doi. org/10.35945/gb.2020.10.010

Chikobava, M. (2020). Metamorphoses of the monetary system against the background of a modern pandemic, The 5th International Scientific Conference, Challenges of Globalization in Economics and Business, Proceedings, 476-486. (In Georgian).

https://www.tsu.ge/assets/media/files/23/\%E1\%83\%A4\%E1\%83\%90\%E1\%83\%98\%E1\%83\%9A\%E1\%83\%94\%E1\%83\%91 \%E1\%83\%98/Programa\%20konferencia.pdf

Durden, T. (2021). Insider-Selling Explodes To Record Highs As Buybacks Re-Emerge. January 20, 2021. https://www.zerohedge.com/markets/insider-selling-explodes-record-highs-buybacks-re-emerge

Financial Times, (2021). Baupost's Seth Klarman compares investors to "frogs in boiling water", https://www.ft.com/content/9c3ecb09-c4bd-4066-a462-af496725105d

Katasonov, V. (2019). What is helicopter money, (in Russian). 27.12.2019.

https://www.fondsk.ru/news/2019/12/27/chto-takoe-vertoletnye-dengi-49778.html

Katasonov, V. (2020). Inclusive capitalism as the ideology of the Great Reset, (in Russian). 21.12.2020. https://www.fondsk.ru/ news/2020/12/21/inkljuzivnyj-kapitalizm-kak-ideologija-velikoj-perestrojki-52523.html

Ossinger, J. (2021). Stimulus Checks Bring Hope for Bull Market Roiled by Bond Yields. March 10, 2021. https://www.bloomberg. com/news/articles/2021-03-10/stimulus-checks-bring-hope-for-bull-market-roiled-by-bond-yields

Papava, V., \& Charaia, V. (2021). The Problem of the Growth of Georgia's Public Debt during the Economic Crisis under the Covid-19 Pandemic. GFSIS Expert Opinion, 152. Tbilisi, Georgian Foundation for Strategic and International Studies. DOI: 10.13140/RG.2.2.10091.57120.

Sedov, D. (2019). American shutdown and its possible consequences (in Russian). 08.01.2019. https://www.fondsk.ru/ news/2019/01/08/amerikanskij-shutdown-i-ego-vozmozhnye-posledstvija-47411.html

Yardeni, E. \& Mali, Q. (2021). Central Banks: Monthly Balance Sheets. Yardeni Research, Inc. Octomber 29, 2021. https://www. yardeni.com/pub/peacockfedecbassets.pdf 